\title{
CARACTERIZAÇÃO DA FROTA PESQUEIRA DE PARINTINS, ITACOATIARA E MANACAPURU, ESTADO DO AMAZONAS.
}

\author{
Vandick da Silva BATISTA ${ }^{1}$
}

\begin{abstract}
RESUMO - A frota pesqueira de Manacapuru, Itacoatiara e Parintins foi analisada em suas características físicas e comparada com a frota Manaus através de dados coletados diretamente nos portos de desembarque e de dados secundários obtidos junto à órgãos federais e a Federação de Pescadores. Foi encontrado que as canoas isoladas variaram de tamanho entre os centros analisados, sendo as maiores encontradas em Manacapuru e Itacoatiara. Os barcos de pesca de Parintins foram significativamente menores que os barcos de pesca de Manaus, Manacapuru e Itacoatiara. O motor Yanmar predominou em todos os municípios, havendo uma relação linear entre a potência deste motor e o tamanho do barco. Manacapuru apresenta barcos mais velhos, seguido da frota de Itacoatiara. As frotas de Parintins e Manaus foram as mais jovens, tendo esta última maior amplitude de idades. Foram encontrados entre 5-6 pescadores por barco de pesca e 2-3 por canoa isolada. A duração das pescarias efetuadas pelos barcos de pesca é crescente descendo o rio, mas não difere para as canoas isoladas entre os centros urbanos. $O$ rendimento da pesca foi menor para os barcos de pesca de Parintins, sendo o rendimento por pescador dos barcos de pesca melhor que o das canoas no período de safra. A partir dos resultados concluiuse que há diferenciação nas características da frota que desembarca em Parintins (Médio Amazonas) em relação aquela desembarcando em Itacoatiara e Manacapuru e que a pesca efetuada com canoas isoladas deve ser tratada diferenciadamente daquela efetuada com barcos de pesca.
\end{abstract}

Palavras-chave: pesca artesanal, frota pesqueira, Amazônia central

\section{Chacterization of the commercial fishing fleet from Amazonas States, Brazil}

\begin{abstract}
The physical characteristics of the Manacapuru, Itacoatiara and Parintins fishing fleets were analyzed and compared with the Manaus fleet, using data collected from the landing ports along with secondary data obtained from federal institutions and the Amazonas Association of Fishermen. It was found that the size of individual canoes varied according to the center analyzed, with the larger ones found in Manacapuru and Itacoatiara. The size of the Parintins fishing vessels was significantly smaller than those in Manaus, Manacapuru and Itacoatiara. Yanmar engines predominated in all municipal districts. There was a linear relationship between the engine power and size of the vessel. The oldest vessels were found in the Manacapuru fleet, followed by the fleet of Itacoatiara. The Parintins and Manaus fleets were the newest, although the latter had the greatest age range. Fishing boats tend usually to have 5 to 6 fishermen and individual canoes 2 to 3 . While fishing vessels working down river make longer trips, no difference in trip length was found for individual canoes in the different municipalities analyzed. Parintins fishing boats produced less fish than those of Manacapuru and Itacoatiara. Yield per fisherman during the height of the fishing season was greater for fishing boats than for individual canoes. Results indicate that the Parintins fleet differs from the others and that the individual canoe fishery must be considered separately from that of the fishing boats with regards to fishery management policy and actions.
\end{abstract}

Key-words: artesanal fishery, fishing fleet, central Amazônia

${ }^{1}$ Professor, Universidade Federal do Amazonas, FCA-DEPESCA, Campus Universitário, Manaus, AM, 69077-000, Brasil E-mail: vbatista@fua.br e tchoni@uol.com.br 


\section{INTRODUÇÃO}

A dinâmica da exploração dos recursos pesqueiros é uma parte fundamental das ciências pesqueiras que têm recebido pouca atenção da pesquisa e portanto pouco tem contribuído para o manejo da pesca (Hilborn, 1985). O pescador comercial é o explorador que faz o elo entre a riqueza dos recursos presentes nos rios e lagos com a população, principalmente a urbana. Sua atividade é fundamental para a nutrição humana da população amazônida (Giugliano et al., 1978; Batista et al., 1998), contribuindo assim para o bem estar social nestes centros, através de sua função ecológica de predador sobre os recursos pesqueiros demandados por estas populações.

O "explorador" ou "predador" pouco tem sido avaliado por cientistas na região (exceções são IPT, 1979 e SUDEPE, 1976), não existindo caracterizações relevantes à sua importante função ecológica. A caracterização do explorador e de seus meios de deslocamento são fundamentais para o manejo da pesca, pois possibilitam ao administrador conhecer e compreender o objeto principal de manipulação. Esta discriminação prejudica e mesmo inviabiliza a administração da atividade pesqueira na Amazônia, assim como tem ocorrido mundialmente por causas diversas (Hilborn \& Walters, 1992).

O propósito geral deste trabalho é gerar informação básica necessária para o estudo da dinâmica da pesca, efetuando a caracterização da frota utilizada pelo pescador que abastece os centros urbanos de médio porte na Amazônia Central, avaliando suas similaridades e diferenças com a frota utilizada pelo pescador que abastece a cidade de Manaus. Neste contexto, podem ser definidas variáveis que afetam a ambos contextos e que podem ser utilizadas em instrumentos administrativos que viabilizem a explotação sustentada dos recursos pesqueiros na região.

\section{MATERIAL E MÉTODOS}

O sistema de estatística pesqueira implantado entre setembro de 1996 e agosto de 1997 nos centros urbanos de Manacapuru, Itacoatiara e Parintins forneceu os dados primários que foram base para este trabalho , assim como foram utilizados dados sobre as características das embarcações obtidas por várias fontes de dados (IBAMA; Capitania dos Portos; Federação dos Pescadores do Amazonas, Roraima e Acre; e cadastro de embarcações FUA/INPA/IBAMA/ CEPNOR).

As informações obtidas na estatística e que foram utilizadas no presente trabalho foram: data da chegada; nome da embarcação; local de pesca e tipo de pesqueiro; número de pescadores; dias pescando; captura por tipo de pescado.

Das informações obtidas sobre as características físicas das embarcações de pesca, foram utilizados: comprimento do barco; ano de construção; marca do motor; e potência (HP).

Os tipos de embarcações que desembarcaram pescado nos três municípios foram canoa, barcos de pesca, barcos de frigoríficos e recreios, sendo analisadas as características dos dois primeiros tipos, os quais são os únicos que saem efetivamente para pescar.

As análise de variância e de covariância, e a análise a posteriori de diferença mínima significativa (LSD), os cálculos de médias, medianas e desvios, assim como o teste não paramétrico de Kruskall-Wallis, foram efetuados no programa STATISTICA, com referenciais teóricos de Sokal \& Rohlf (1981) e Tabachnick \& Fidell (1996).

\section{RESULTADOS}

\section{Distribuição de tamanho das embarcações de pesca}

A frota pesqueira avaliada, apresenta dois tipos básicos de embarcações que pescam: a canoa isolada e os barcos de pesca, sendo que este também pesca a partir de canoas, porém em atividades que envolvem frequentemente mais de uma unidade.

As canoas isoladas variaram de tamanho entre os centros analisados, sendo que em Parintins apresentaram tamanho modal na classe de 2-3 m, a qual incluiu mais de 50\% 
das embarcações registradas (Fig. 1). Manacapuru e Itacoatiara apresentaram canoas maiores, respectivamente com modas entre as classes 5-6 m e 6-7m, não havendo diferença significativa entre ambas (teste de KruskallWallis $\mathrm{P}>0,05$ ). Parintins apresentou tanto a menor canoa quanto uma das maiores, respectivamente com $1,7 \mathrm{~m}$ e $14 \mathrm{~m}$, sendo que em Itacoatiara não foram registradas canoas menores que $2 \mathrm{~m}$ ou maiores que $10 \mathrm{~m}$, mesmo com o maior número de unidades discriminadas. Em Manacapuru foram acompanhadas poucas canoas no desembarque, o que prejudicou a representatividade da amostra. Mesmo assim foram encontradas canoas entre 5 e $14 \mathrm{~m}$, sendo que o limite inferior real deve cair para $2 \mathrm{~m}$ com uma amostragem maior, segundo informações de pescadores locais.

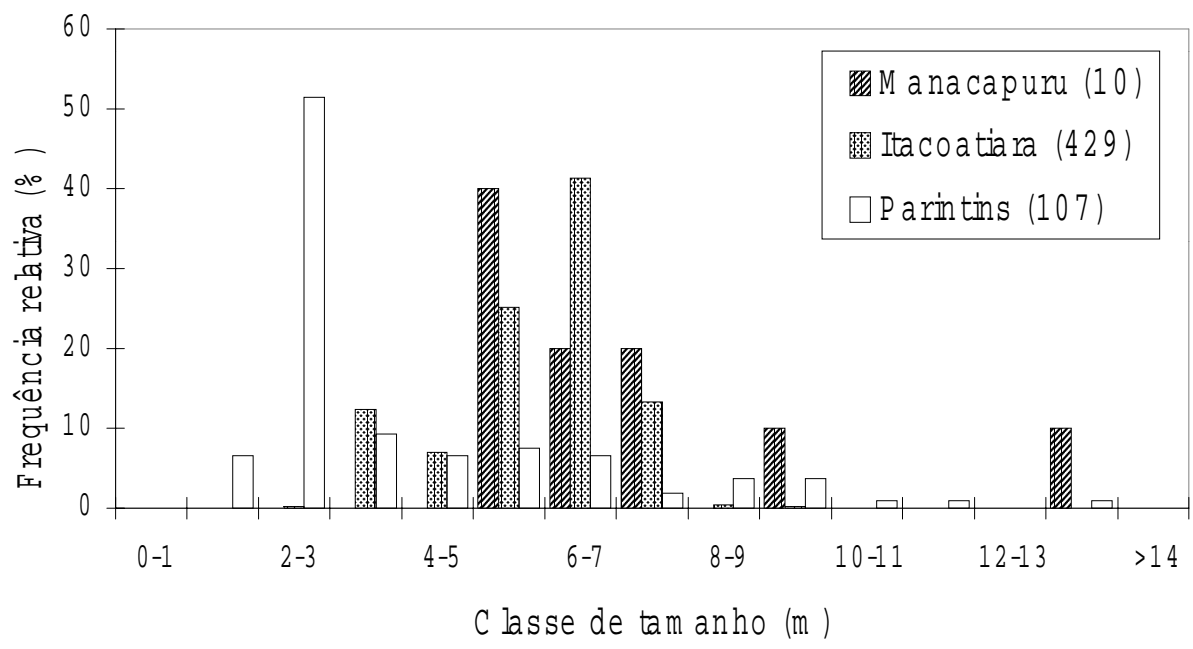

Figura 1 - Distribuição relativa de comprimento de canoas com pescado para venda nos centros urbanos de Manacapuru, Itacoatiara e Parintins. Os números entre parênteses indicam o número total de canoas amostradas.

Os barcos de pesca apresentaram comprimento do casco entre 8 e $24 \mathrm{~m}$ em Manacapuru, entre 9 e 23 em Itacoatiara e entre 4 e $20 \mathrm{~m}$ em Parintins (Fig. 2). As informações referentes a frota de Manaus foram incorporadas, registrando-se que a média do tamanho das embarcações foi homocedástico entre níveis $(\mathrm{P}>0,05)$, não havendo nenhuma distribuição que tenha se desviado notoriamente da normalidade. Assim, foi efetuada a análise de variância, obtendo-se que há diferenças significativas ao nível de 5\% (Tabela 1). A análise a posteriori por diferença mínima significativa, indicou que as embarcações de Parintins foram significativamente distintas dos barcos de pesca dos outros centros urbanos (Tabela 2), sendo que as embarcações das outras cidades não apresentaram diferença entre si.

\section{Motor e potência}

Em Manacapuru 90\% das embarcações possuíam motores da marca Yanmar, sendo os restantes distribuídos assim: 3\% Kubota, 3\% CMC, 2\% Yamaha e 2\% MTU. Em Itacoatiara o predomínio dos motores Yanmar se mantêm, mas reduz-se para $85 \%$ dos casos, os barcos restantes apresentaram 7\% de MWM, 4\% de Daya e 4\% de CMC. Em Parintins a participação dos Yanmar cai para $82 \%$ do total de barcos com informações, havendo também maior variedade nos restantes, destacando-se os MWM com 11\% do total, havendo ainda 7\% igualmente divididos entre as marcas Tobatta, Mercedes, Daya e CMC. 


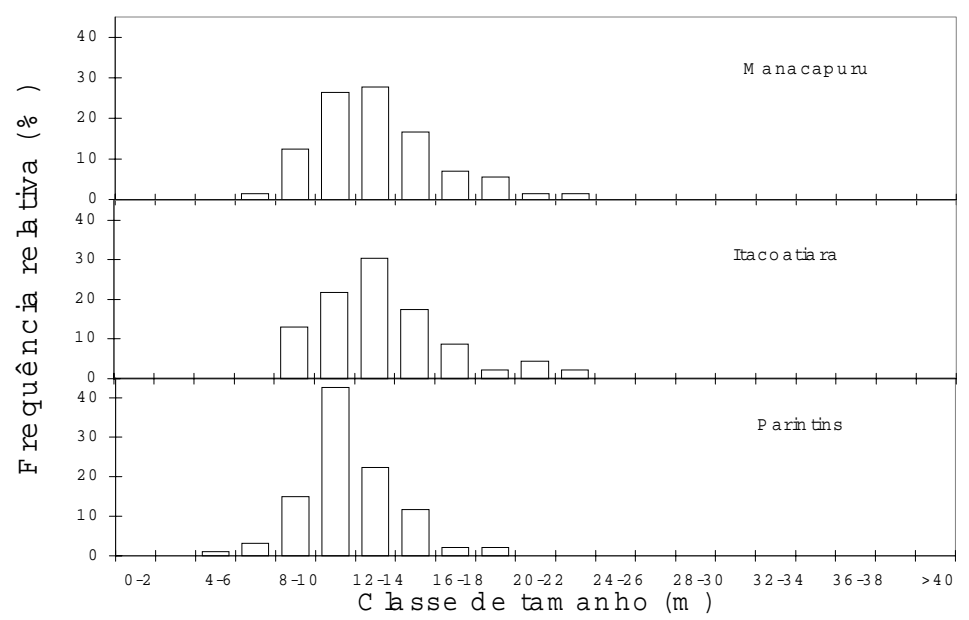

Figura 2 - Distribuição de comprimento de uma amostra de barcos de pesca que desembarcaram em Manacapuru, Itacoatiara e Parintins entre 1996 e 1997.

Tabela 1 - Análise de variância do comprimento médio dos barcos de pesca em relação ao fator Cidade.

\begin{tabular}{lcccc}
\hline $\begin{array}{l}\text { Fonte de } \\
\text { variação }\end{array}$ & $\begin{array}{c}\text { Graus de } \\
\text { liberdade }\end{array}$ & $\begin{array}{c}\text { Quadrado } \\
\text { médio }\end{array}$ & $\mathrm{F}$ & $\mathrm{P}$ \\
\hline Cidade & 3 & 90.79253 & 9.228863 & $<0,01$ \\
Erro & 443 & 9.837889 & & \\
\hline
\end{tabular}

Tabela 2 - Significância do teste de contraste comprimento médio dos barcos de pesca, efetuado através da diferença mínima quadrada (LSD) entre os níveis que formam o fator Cidade. Abaixo da diagonal, estão indicados em cinza as células com contraste significativo $(\mathrm{P}<0,05)$.

\begin{tabular}{lccccc}
\hline Cidade & & $\{$ MCP & $\{$ ITA $\}$ & $\{$ PAR & $\{$ MAN \\
& & 13,309 & 13,723 & 12,063 & 14,112 \\
\hline Manacapuru & $\{$ MCP & & 0,485 & 0,013 & 0,057 \\
Itacoatiara & $\{$ ITA & 0,485 & & 0,004 & 0,441 \\
Parintins & $\{$ PAR & 0,013 & 0,004 & & $<0,001$ \\
Manaus & $\{$ MAN & 0,057 & 0,441 & $<0,001$ & \\
\hline
\end{tabular}

A potência do motor em HP variou de acordo com a marca. Assim, diferenciamos a marca predominante Yanmar das restantes no diagrama de dispersão do comprimento do barco (CB) versus HP (Fig. 3), observando que a distribuição é dispersa para motores de outras marcas, não sendo apropriado estimar um modelo empírico para as marcas separadamente, visto o baixo número de dados nestes casos. Para os motores Yanmar observou-se que a relação é linear, como já foi observado para a frota desembarcando em Manaus. Uma análise de covariância foi efetuada para o fator Cidade, após verificar o paralelismo entre as retas $\left(\mathrm{F}_{3,429}=0,727 ; \mathrm{P}>0\right.$, 537), encontrando que as retas não foram significativamente distintas (Tabela 3), sendo então calculada a equação comum à frota pesqueira:

$$
\begin{aligned}
& \mathrm{HP}=-32,4137+4,2755 * \mathrm{CB} \\
& \mathrm{r}^{2}=0,6054 \quad \mathrm{~F}_{1,435}=667,43 \quad \mathrm{P}<0,01
\end{aligned}
$$




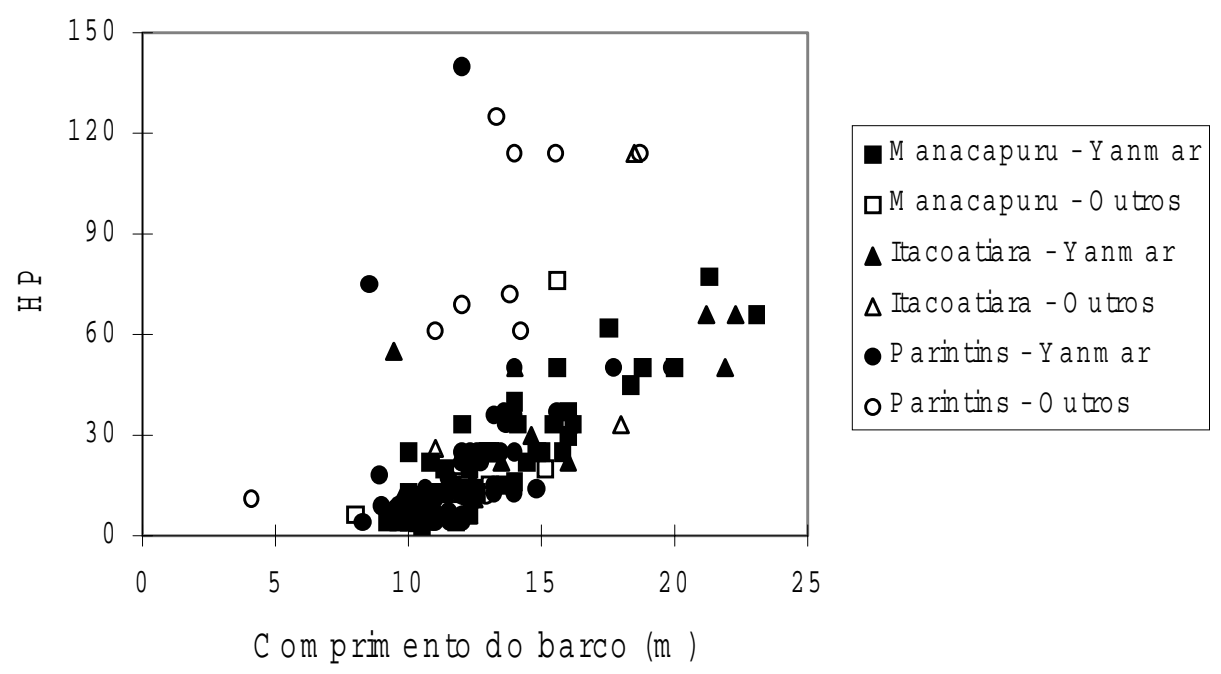

Figura 3 - Variação da potência do motor em HP em relação ao comprimento do barco para os barcos desembarcando pescado nas cidades de Manacapuru, Itacoatiara e Parintins. Os símbolos preenchidos referem-se a motores Yanmar e os vazios às marcas restantes.

Tabela 3 - Análise de variância do fator Cidade, tendo como covariante o comprimento do barco para a análise de covariância da variável HP à frota pesqueira com motores Yanmar desembarcando em cidades do interior.

\begin{tabular}{ccccc}
\hline Fonte de variação & Graus de liberdade & Quadrado médio & $F$ & $P$ \\
\hline Cidade & 3 & 92,4143 & 0,7222 & $>0,54$ \\
Erro & 432 & 127,9602 & & \\
\hline
\end{tabular}

\section{Estrutura de idade das embarcações}

Tomando 1997 como ano base para cálculo da idade, os barcos de pesca de Manacapuru apresentavam idades entre 5 e 33 anos, de Itacoatiara entre 3 e 26 anos, e Parintins entre 1 e 30 anos (Fig. 4), sendo que as distribuições foram diferentes entre os três centros. A comparação não paramétrica das distribuições pelo teste de Kruskall-Wallis indicou diferenças significativas $(\mathrm{P}<0,05)$. A análise das medianas e dos quartis indica que a frota de Manacapuru apresenta barcos mais velhos (Fig. 5), seguido da frota de Itacoatiara. As frotas de Parintins e Manaus apresentam grande similaridade na idade da frota, embora esta última apresente maior amplitude devido a presença de embarcações mais velhas.

\section{Número de pescadores e dias pescando}

Os barcos de pesca que desembarcaram em Manacapuru e Parintins apresentaram distribuição similar do número de pescadores embarcados por pescaria (Fig. 6), variando de 2-3 a 14-15 pescadores, e modas principais entre 3 e 6 pescadores, sendo que a moda em Manacapuru esteve na classe de 6 pescadores (média de 5,7 pescadores) e a de Parintins na classe de 5 pescadores (média de 4,7 pescadores). Itacoatiara apresentou a mesma amplitude, porém distribuição distinta, concentrando mais de $40 \%$ das pescarias com 6 pescadores (média geral de 6,1).

As canoas isoladas apresentam modas fortes nas três cidades, sendo que em Itacoatiara 


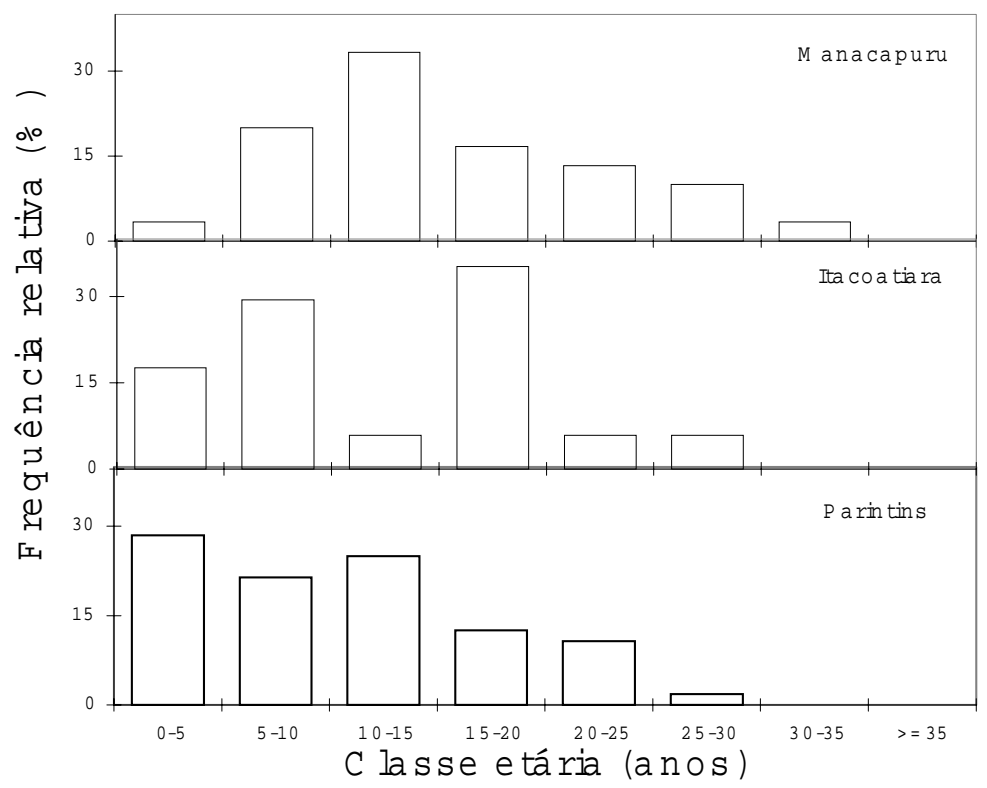

Figura 4 - Distribuição etária de barcos de pesca desembarcando nas cidades de Manacapuru, Itacoatiara e Parintins entre setembro de 1996 e agosto de 1997.

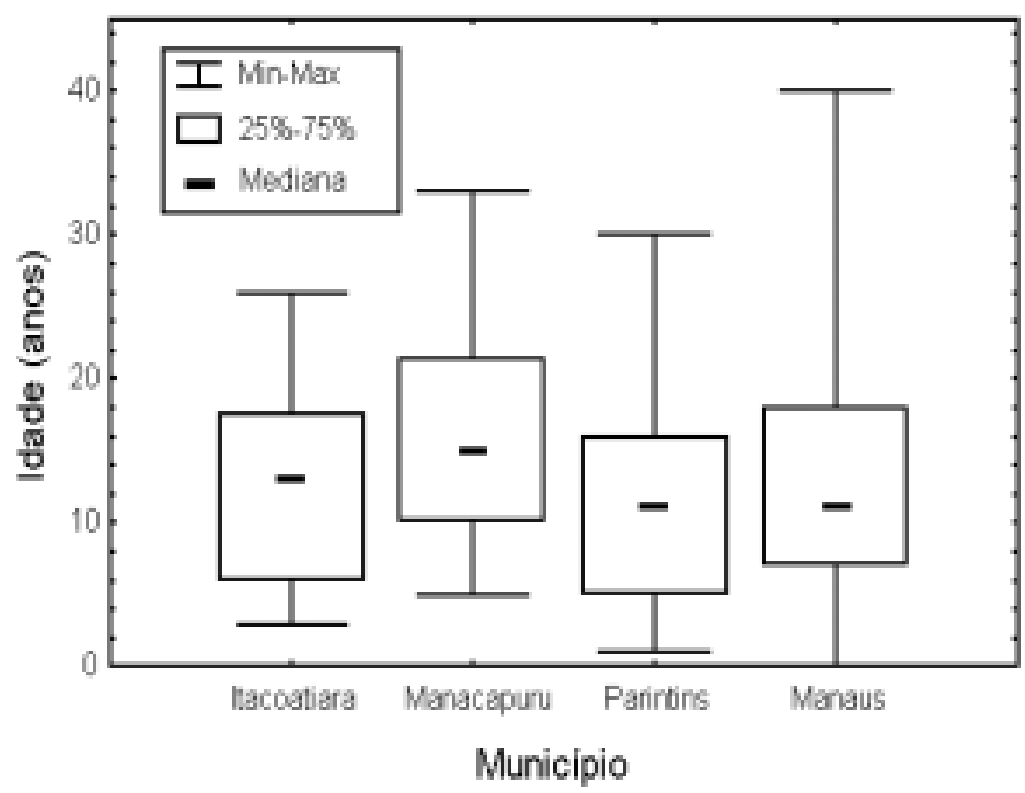

Figura 5 - Medianas, 25 e 75 quartis e da amplitude etária da frota desembarcando em três cidades de porte médio da Amazônia Central. 


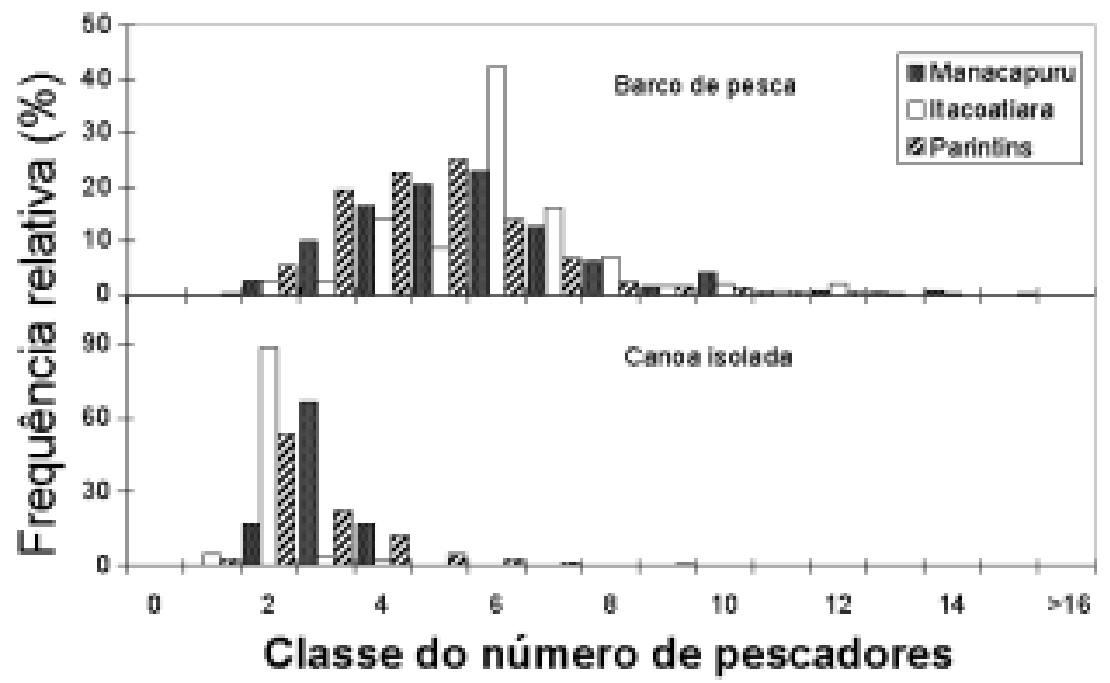

Figura 6 - Distribuição da frequência relativa do número de pescadores por barco de pesca (acima) e canoa isolada (abaixo) nas cidades de Manacapuru, Itacoatiara e Parintins.

e Parintins predominam dois pescadores por pescaria e em Manacapuru predominam 3 pescadores por pescaria. O número mínimo é de um pescador por canoa, sendo que em Manacapuru este valor foi informado por vários pescadores locais. O número máximo usualmente é de quatro pescadores, porém em Parintins chegaram a ser registrados até 9 pescadores em canoas isoladas.

O número de dias pescando variou desde algumas horas de pesca (em Itacoatiara) ou um dia de pesca (mais de $15 \%$ das pescarias nos três centros), até 20 dias de pesca, tendo ocorrido três registros isolados em Manacapuru e Itacoatiara que apresentaram 30, 50 e 60 dias pescando, sendo considerados excepcionais (Fig. 7). Mais de $50 \%$ das pescarias foram realizadas em até 6 dias de pesca, com percentual crescente a medida em que se vai descendo o rio, sendo de 55,6\% em Manacapuru, de 63,5\% em Itacoatiara e de $85,1 \%$ em Parintins. As canoas isoladas estiveram pescando entre algumas horas até 3 dias em Itacoatiara, sendo que em Parintins a pesca foi mais longa, atingindo até 5 dias, mas a moda esteve em apenas um ou dois dias (cerca de $88 \%$ do total de casos).

\section{Produtividade entre tipos de embarcações que pescam}

Os pescadores de ambos tipos de embarcações, utilizam o mesmo tipo de meio de deslocamento na dinâmica da pesca, que é a canoa, porém a existência do barco de pesca permite transporte de apetrechos de maior tamanho, com mais facilidade e com maior mobilidade na região, podendo apresentar produtividade distinta. Observa-se que o rendimento diário durante o final da vazante, seca e início da enchente é bastante similar entre pescadores de canoas isoladas e os pescadores de barcos de pesca (Fig. 8), sendo exceção em Itacoatiara, onde os pescadores de canoas isoladas apresentaram maior rendimento até o início da enchente. A partir de dezembro observamos nos registros de todas as cidades, que ocorreu um gradual e crescente aumento no rendimento dos pescadores baseados nos barcos de pesca, atingindo um máximo entre maio e junho (pico da cheia), enquanto que os pescadores de canoas isoladas mantêm rendimentos estáveis, porém relativamente bem inferiores. 




Figura 7 - Distribuição da frequência relativa do número de dias pescando registrado com a frota desembarcando com barcos de pesca (acima) e canoas isoladas (abaixo) nas cidades de Manacapuru, Itacoatiara e Parintins.

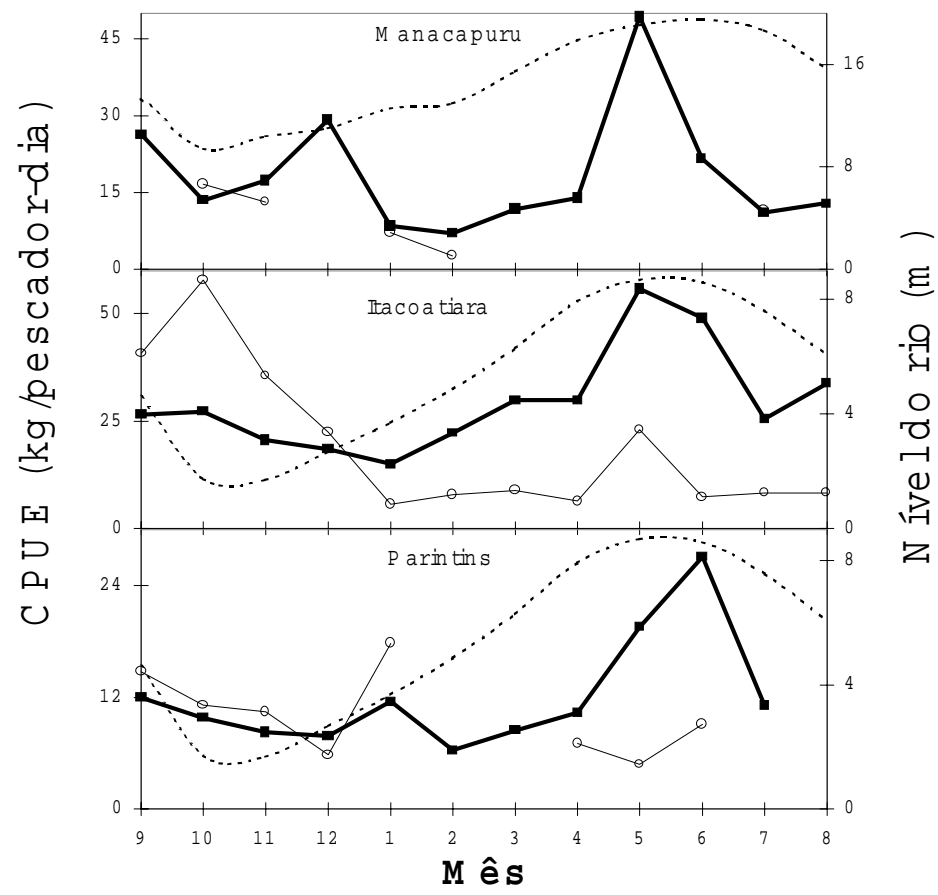

Figura 8 - CPUE em kg/pescador-dia de barcos de pesca (linha grossa) e de canoas isoladas (linha fina) que desembarcaram em Manacapuru, Itacoatiara e Parintins ao longo de um ano. A linha pontilhada indica a variação do nível do rio na estação fluviométrica mais próxima. 


\section{DISCUSSÃO}

A capacidade de pesca de uma frota pode ser dimensionada a partir de quatro componentes básicos: sua eficiência técnica, o número de barcos de pesca, o tamanho de cada embarcação e pelo tempo que cada unidade gasta pescando (Smith \& Hanna, 1990). Particularmente a eficiência técnica é muito difícil de ser estimada e monitorada (Hilborn \& Ledbetter, 1985; Shardlow, 1993), havendo variações nas estratégias de busca do peixe, as quais têm sido avaliadas por uma perspectiva pesqueira (e.g. Hilborn \& Ledbetter, 1979; Hilborn \& Walters, 1987; Vignaux, 1996) e por uma perspectiva antropológica (e.g. McCay, 1978; Smith \& McKelvey, 1986; Smith \& Hanna, 1990).

Existe ainda pouca informação em geral neste âmbito, sendo mais usual associar o poder de pesca com medidas estáticas, como o tamanho das embarcações e a potência de motores, o que permite preencher as necessidades básicas de informação em várias pescarias (e.g. Palsson \& Durrenberger, 1982; Irusta, 1997). Porém, em vários casos estas características não bastam para explicar as diferenças de rendimento observadas entre embarcações pesqueiras (Hilborn \& Ledbetter, 1985). Número de barcos, o tamanho dos barcos e pelo tempo de pesca são itens mais fáceis de acompanhamento, porém a nível regional nem mesmo estes têm recebido uma atenção mínima necessária que permita embasar o planejamento das atividades de manejo da pesca na região com informações confiáveis.

Cada um destes fatores apresenta uma demanda diferenciada de informação, cuja qualidade e disponibilidade é altamente variável a nível mundial e muito pouco conhecida a nível da região amazônica. $\mathrm{O}$ perfil estrutural da frota, onde o presente trabalho se situa, é assim prioritário para o desenvolvimento em curto prazo de estratégias para a administração pesqueira em uma região, básico para poder estabelecer avaliações mais refinadas.

A informação aqui analisada é o início de um processo que deve se consolidar para que sejam disponibilizados dados mais precisas sobre a realidade existente. Logo, é necessário enfatizar que as generalizações aqui efetuadas devem ser tomadas de forma conservadora, até confirmação por meio de estudos dirigidos.

Neste contexto, observou-se que a diferença na magnitude do tamanho das embarcações de pesca das cidades do interior em relação a Manaus não é pronunciada, sendo que apenas a frota de Parintins apresentou-se distinta das demais $(9 \%$ a $15 \%$ menor em média). As canoas isoladas de Parintins foram notoriamente menores que as registradas em Itacoatiara e Manacapuru, que foram similares entre si. Em Santarém, Isaac et al. (1998) informam que os barcos de pesca daquela cidade apresentam em torno de $10,2 \mathrm{~m}$ de comprimento, o que os discriminaria da frota de Manaus-Manacapuru-Itacoatiara, mas que poderiam ser similares aos de Parintins dependendo da dispersão dos dados. De toda forma, pela ordem dos valores encontrados, há indícios de que haveria uma diminuição do tamanho dos barcos de pesca entre Manaus e Santarém, o que pode estar relacionado com as características distintas da morfologia da bacia hidrográfica na Amazônia Oriental em relação a Amazônia Central. Entretanto a isto pode estar associada uma variação de técnicas de pesca que demandam um meio de transporte diferenciado. Exemplo disto foi observado por Batista (1998), onde é apresentado que a participação da redinha nas pescarias é menor em Parintins, sendo que a redinha demanda a participação de canoas de maior porte. As canoas isoladas também foram menores em Parintins o que generalizaria o padrão observado, mas não há informação sobre outros centros, assim é mais adequado manter esta questão em aberto até ampliar as informações a nível geográfico.

As cidades do interior e Manaus apresentaram uma relação similar da potência do motor com o tamanho da embarcação, sendo que parte dos $40 \%$ de variação restante deve estar relacionado ao retardo com que ocorre a adequação da potência do motor para novas embarcações (Batista, 1998). Isto se deve ao fato que na aquisição de um novo barco o armador prefere investir em um casco maior, podendo deslocá-lo com uma máquina de menor potência que usualmente pertenceu a outro barco menor de sua propriedade, trocando o motor mais tarde após se capitalizar, o que 
pode demorar. Interessante porém é a participação decrescente dos motores Yanmar a medida em que se distancia de Manaus, embora seja necessário avaliar a situação no Pará para confirmar esta tendência, o que é potencialmente importante para trabalhar posteriormente com a questão da eficiência do deslocamento e a adequação da potência instalada para o uso atualmente observado na atividade pesqueira.

O aparecimento e a retirada de embarcações da frota são equivalentes à natalidade e mortalidade de um predador, sendo importantes para o entendimento da dinâmica do sistema. Dentro do tópico natalidade, a única informação disponível foi tomada em Manaus (Batista, 1998), apresentando um acréscimo estimado em cerca de 24 barcos por ano. Entretanto não têm sido tomadas informações sobre os meios de financiamento que viabilizaram a construção dos barcos ou os fatores motivadores que determinaram a decisão deste investimento, o que deve ser efetuado para viabilizar uma utilização mais apropriada desta informação. Da mesma forma, a saída de barcos da pesca de Manaus já foi evidenciada (Batista, 1998), entretanto sem informações sobre a razão da saída. Hilborn \& Walters (1992) indicam que há perdas de embarcações na pesca por afundamento, depreciação física seguida de abandono, mudança de atividade e mudança de área de atuação na pesca, parecendo ser mais importantes o segundo e terceiro itens na região, segundo pescadores entrevistados.

Para a frota das cidades do interior no Amazonas, a dinâmica deve ser mais complexa, por estar sujeita a influência do grande centro consumidor (Manaus) e sua demanda, o que explicaria a variação nas distribuições etárias observada nas mesmas, tais como a frota significativamente mais velha de Manacapuru, cidade que parece concentrar várias embarcações antigas que deixaram de frequentar normalmente o porto de Manaus. Também deve ser importante a existência de meio de financiamento, visto que o capitalizador atual da pesca em Manaus é o despachante (Parente, 1985), o qual não está presente ou tem menor importância nas cidades analisadas.

Outra questão polêmica e de grande importância é avaliar a atividade das embarcações pesqueiras como fonte geradora de trabalho e de ocupações, visto que o conceito de emprego apenas é útil no contexto dos frigoríficos e do comércio nos centros urbanos. Neste ponto, tomando-se o tamanho da frota indicado por Batista (1998) e o número médio de pescadores trabalhando nos barcos de pesca, pode-se dimensionar a magnitude de 20 a 25 mil pescadores profissionais atuando na região.

A eficiência relativa é usualmente muito similar entre o pescador de canoas isoladas e o pescador dos barcos de pesca, porém em todos os centros observamos que há um aumento na produtividade do pescador dos barcos de pesca durante a safra de jaraquis na cheia (Batista, 1998). Nesta pescaria, o tempo gasto antes da captura para encontrar o cardume é normalmente elevado e o tempo de captura é pequeno. Uma vez encontrado o cardume o pescador dos barcos de pesca pode descarregar toda a produção excepcional no barco em que está baseado várias vezes, enquanto que o barco de pesca vai acompanhando o cardume. O pescador de canoa isolada gasta tempo buscando o cardume, mas uma vez que o encontre, atinge um ponto de saturação rapidamente e tem que ir ao mercado para vender o pescado. Feito isto, tentaria retornar para o cardume, o que não ocorre pois o dia termina, levando-o a realizar apenas um lance no dia, que é a sua rotina de pesca ao longo do ano. Já o pescador do barco de pesca, faz vários lances no mesmo período sem perder o cardume. A segunda vantagem é trabalho mais coletivo dos barcos de pesca, e a possibilidade de variação nas estratégias de captura conforme o tamanho do cardume, incluindo a união de redes, o trabalho conjunto de duas ou mais canoas, entre outras. O pescador isolado não é flexível neste sentido, embora seja um especialista em determinada área, o que permite que explore estas áreas mais eficientemente principalmente durante a seca, quando consegue detectar melhor os locais onde os peixes ficaram barrados, além de conhecer melhores acessos para alcançar estes pesqueiros e para escoar a produção deles.

Dentro de uma perspectiva de manejo, as frotas baseadas no interior apresentam uma vantagem importante; seus tripulantes são conhecidos e suas lideranças estão mais 
próximas para negociar com outros interessados na questão a nível local, como as comunidades ribeirinhas, a prefeitura municipal, o posto do IBAMA (Agência Federal do Meio Ambiente), do IDAM (Secretaria de Produção do Governo do Estado), etc. Também devem perceber melhor as mudanças no ambiente local que influem sobre a abundância dos recursos pesqueiros, tornando-os mais dependentes da conservação do sistema, visto que são mais dependentes da disponibilidade local dos recurso, não se deslocando muito para fora da área de pesca tradicional.

Outro aspecto importante é que estas frotas locais produzem pescado e capital que beneficiam também a sede municipal e as pequenas vilas locais, e não apenas Manaus, o que pode facilitar a negociação com a sociedade local sobre estratégias de conservação do ambiente e dos recursos. $\mathrm{O}$ perfil desta frota pode ser mantido, considerando a baixa autonomia que cada unidade dispõe, a qual as limita a um raio de ação restrito, porém há deficiências técnicas óbvias em muitas embarcações e que devem receber atenção especial por reduzirem a rentabilidade do setor. Problemas com conservação do pescado também tendem a ser menos graves, visto que o tempo de pesca é bem menor que o da frota de Manaus (Batista, 1998).

As formas de escoamento da produção e a negociação do acesso as áreas de pesca parecem ser problemas maiores e que devem ser pontos importantes na pauta de atividades de manejo da atividade pesqueira em cidades do interior do Amazonas, devendo porém haver maior aprofundamento na caracterização técnica aqui iniciada.

\section{AGRADECIMENTOS}

Agradecemos à SUDAM, FNMA, Fundação Banco do Brasil e CNPq pelo apoio financeiro; ao IBAMA-SUPES/AM, ao Grupo Executivo do Setor Pesqueiro e à Federação de Pescadores do Amazonas e Roraima pela gentil cessão de dados; e à Francisca Cavalcante da Silva, Ivanildo Lima Alves dos Santos e Herberth Franco Siqueira pelo valioso apoio técnico.

\section{BIBLIOGRAFIA CITADA}

Batista, V.S. 1998. Distribuição, dinâmica da pesca e dos recursos pesqueiros na Amazônia Central. Tese de doutorado, INPA/FUA; Manaus, $291 \mathrm{pp}$.

Batista, V.S.; Inhamuns, A.J.; Freitas, C.E.C. \& Freire-Brasil, D. 1998. Characterization of the fishery in river communities in the low-Solimões/high-Amazon region. Fisheries Management and Ecology, 5, 419-435.

Giugliano, R.; Shrimpton, R.; Arkcoll, D.B.; Giugliano, L.G. And Petrere Jr., M. 1978. Diagnóstico da realidade alimentar e nutricional do Estado do Amazonas. Acta Amazonica, 8(Supl.2), 54 pp.

Hilborn, R.; Ledbetter, M. 1979. Analysis of the British Columbia salmon purse seine fleet: dynamics of movement. Journal of the Fisheries Research Board of Canada, 36, 384-391.

Hilborn, R.; Ledbetter, M. 1985. Determinants of catching power in the British Columbia salmon purse-seine fleet. Canadian Journal of Fisheries and Aquatic Sciences, 42, 51-56.

Hilborn, R.; Walters, C.J. 1987. A general model for simulation of stock and fleet dynamics in spatially heterogeneous fisheries. Canadian Journal of Fisheries and Aquatic Sciences, 44, 1366-1369.

Hilborn, R.; Walters, C.J. 1992. Quantitative fisheries stock assessment, Chapman and Hall, 570 pp.

Hilborn, R.. 1985. Fleet dynamics and individual variation: why some people catch more fish than others. Canadian Journal of Fisheries and Aquatic Sciences, 42, 2-13.

IPT 1979. Avaliação da atual frota pesqueira amazonense e proposição de novas concepções. Relatório parcial $1^{\circ}$ volume. IPT/SICCT/SETRAM, Manaus. 219 pp.

Irusta, C.G.; Aubone, A.; Simonazzi, M.; Ibañez, P. 1997. Estimaciones de los poderes de pesca relativos de la flota de altura convencional merlucera argentina. 
Zona Patagonica: $41^{\circ}-48^{\circ}$ S. INIDEP Informe Técnico, 17, $24 \mathrm{pp}$.

Isaac, V. J., Ruffino, M. L., Milstein, A. 1998. Fisheries ecology in the Lower Amazon: A typical artisanal practice in the tropics.. Ecotropica, 4, 99 - 114.

McCay, B.J. 1978. Systems ecology, people ecology and the anthropology of fishing communities. Human Ecology, 6, 397-422.

Palsson, G.; Durrenberger, P. 1982. To dream of fish: the causes of Icelandic skippers fishing success. J. Anthropol. Res., 38, 227-242.

Parente, V.M. 1995. A economia da pesca em manaus: Organização da produção e da comercialização. Dissertação de mestrado, UFRRJ, $178 \mathrm{pp}$.

Shardlow, T.F. 1993. Components analysis of a density-dependent catchability coefficient in a salmon hook and line fishery. Canadian Journal of Fisheries and Aquatic Sciences, 50, 513-520.

Smith, C.L.; Hanna, S.S. 1990. Measuring fleet capacity and capacity utilisation. Cana- dian Journal of Fisheries and Aquatic Sciences, 47, 2085-2091.

Smith, C.L.; McKelvey, R. 1986. Specialist and generalist: roles for coping with variability. North American journal of Fisheries Management, 6, 88-99.

Sokal, R.R.; Rohlf, F.J. 1981. Biometry. The principles and practice of statistics in biological research. W.H. Freeman and Company, New York, 859.

SUDEPE 1976. Terminal pesqueiro de Manaus. Volume 1. Relatório SUDEPE/ Governo do Amazonas/Prefeitura de Manaus/SUFRAMA, $317 \mathrm{pp}$.

Tabachnick, B.G.; Fidell, L.S. $1996 . \quad$ Using multivariate statistics. HarperCollins College Publishers Inc., New York, $880 \mathrm{pp}$.

Vignaux, M. 1996. Analysis of vessel movements and strategies using commercial catch and effort data from the New Zealand hoki fishery. Canadian Journal of Fisheries and Aquatic Sciences, 53, 2126-2136. 